\title{
La Concepción (Marbella): inicios de la industrialización andaluza
}

\author{
Óscar Gil Delgado | arquitecto, Universidad de Sevilla \\ Juan Carlos Jiménez Barrientos | arqueólogo, Consejería de Cultura \\ URL de la contribución <www.iaph.es/revistaph/index.php/revistaph/article/view/3968>
}

\section{RESUMEN}

En el siglo XIX hay una "anticipación andaluza" en la producción sidero metalúrgica con La Concepción y El Ángel, Marbella (Málaga), donde se establecen los primeros altos hornos indirectos tipo inglés. La acumulación originaria de capital para estos establecimientos radica en su mayor parte en la explotación y agotamiento del mineral de grafito de la sierra marbellí, por parte de Manuel Agustín Heredia. A este primer impacto hay que unir el del bosque y matorral mediterráneo usado como carbón leña combustible en los altos hornos. Sin embargo nunca se resolvió el problema esencial: que la naturaleza del mineral de hierro explotado también en las minas de Marbella tenía un alto contenido en magnetita que requería carbón mineral para su fusión y afino. Esto condujo al establecimiento de una segunda factoría en Málaga capital: La Constancia; La Concepción fundía y La Constancia afinaba. Pero ni aún así se resolvía el problema de los costos de transporte del carbón, lo que llevó al fin de los Altos Hornos de Marbella, pionero del alto horno inglés en España. A partir de mediados-finales del siglo XIX la decadencia de la fundición llevó a un período de actividad extractiva, explotado por compañías inglesas, y que aceleró modificaciones urbanísticas y de infraestructura, como la creación de la línea férrea minera de San Juan, unos almacenes en el casco urbano, y un puente-embarque de hierro que se introducía en el mar.

\section{Palabras clave}

Altos Hornos | Arquitectura industrial | Benahavís (Málaga) | Ferrocarril | Fundiciones | Grafito | Hierro | Marbella (Málaga) | Minería | 


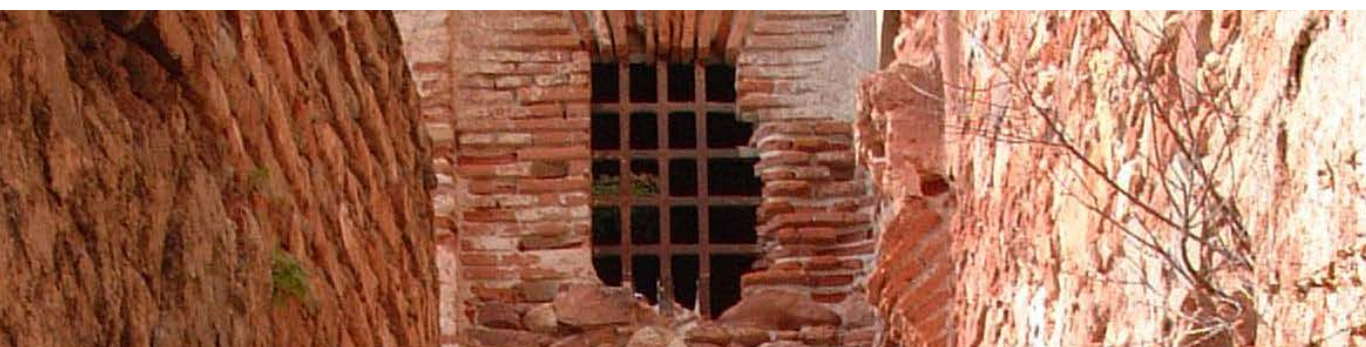

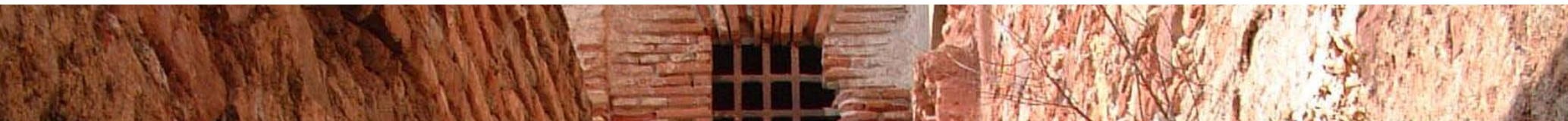

6.5.

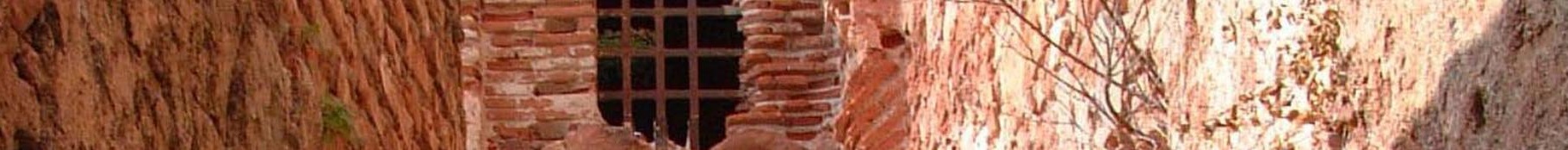

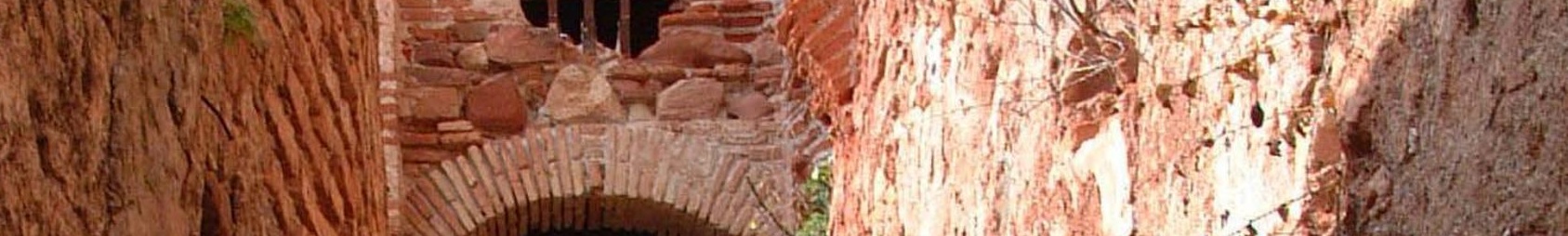

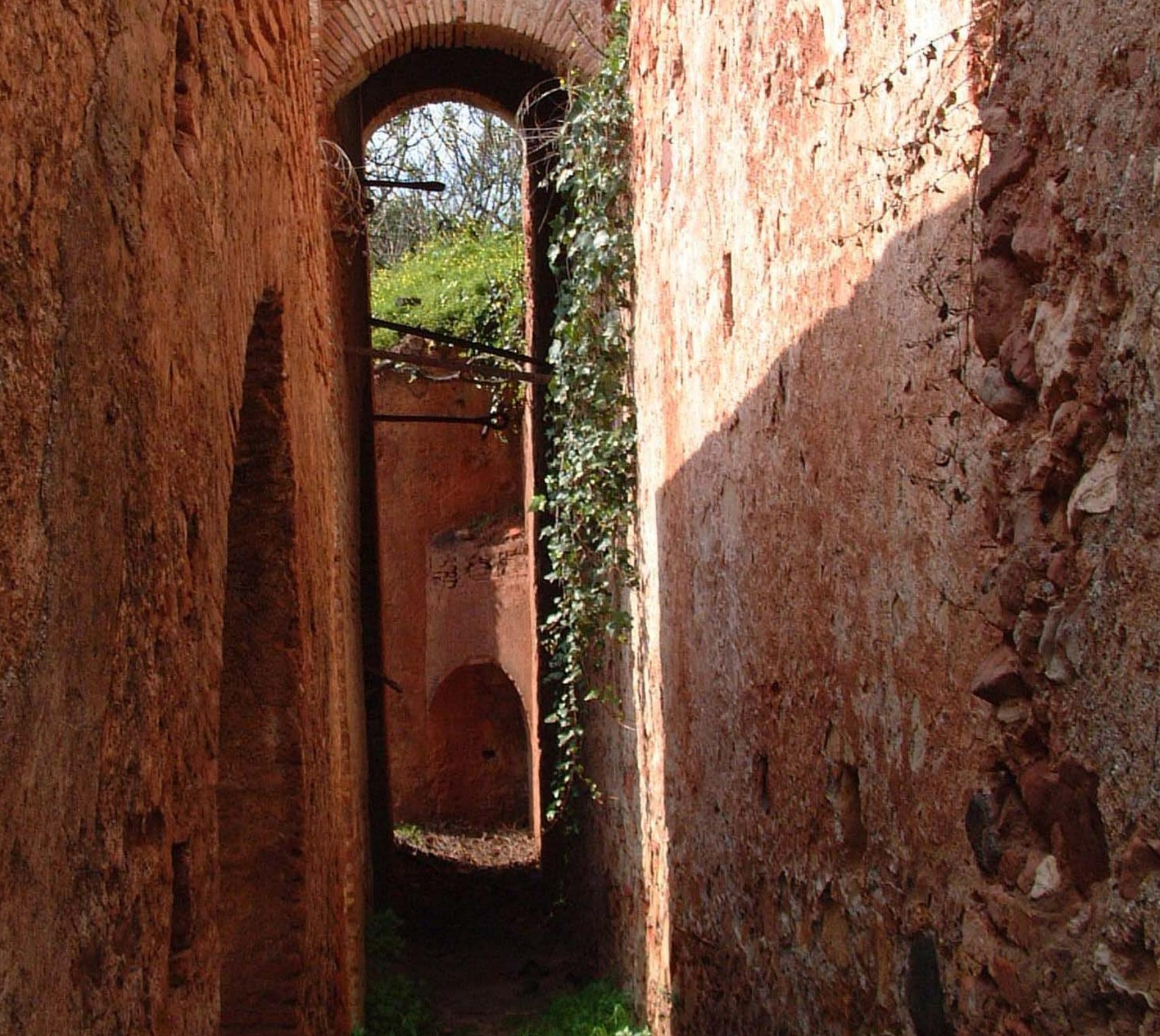


Durante el siglo XIX se abre un nuevo episodio de la "anticipación andaluza" en la producción sidero metalúrgica española; si el primero había tenido lugar en las Reales Fábricas de Cádiz, Málaga y Almería, este segundo vuelve a repetir suelo de extracción-producción en Málaga, e incorpora Sevilla (EI Pedroso); tan sólo La Concepción y El Ángel en Marbella (Málaga) producen el $72 \%$ del hierro español de 1844.

Varias son las circunstancias que sustentan este momento industrial. La primera de ellas la "acumulación originaria de capital" que procede de la explotación minera en una coyuntura mercantil favorable; la explotación del grafito para la fabricación de crisoles, extraído de los criaderos de Cerro de Natías (Benahavís), Igualeja y Pujerra (Málaga). G. Bowles, naturalista irlandés, recorrió España por encargo real reflejando en su informe la existencia en territorio malagueño de molybdana o lápiz de dibujar, y que los criaderos marbellíes estaban muy descuidados, pero años antes desde un consulado extranjero se había obtenido permiso real para explotar 250 quintales anuales. Desde 1749 vecinos de Júzcar (Málaga) extraen el grafito marbellí, en todas sus variantes: haba, perdigón y polvo. A continuación hay intentos de transformarlo in situ y en $1804 \mathrm{E}$. Schnelbenbühel, el conde de Teba, el duque del Infantado, Dionisio Sancho y Gryvegnee crean una sociedad para la fabricación de crisoles. Pero el verdadero momento será el saqueo del grafito durante 1812-13 a manos de Manuel Agustín Heredia quien esquilma la sierra marbellí para proveer grafito para crisoles de proyectiles a ambos bandos; fundamentalmente será en la sierra de La Mora, cordillera divisoria entre los ríos Guadalmedina-Guadalmansa de donde se extrae con agotamiento de los criaderos el grafito marbellí; es el primer gran impacto sobre la serranía pero no el único.

Finalizada la Guerra de Independencia española, M. A. Heredia vuelve a las actividades comerciales anteriores consistentes en el ciclo de la jábega, exportación mediterránea malagueña de almendras, pasas y vino, y regreso desde Europa Central con bienes de equipo. Este ciclo caracterizaba la economía de la capital malagueña desde el siglo XVIII y había atraído a la ciudad a extranjeros europeos y nacionales desde el norte español, como el caso del propio M. A. Heredia (Rabanera de Cameros, La Rioja, 1786). Reanudada la actividad comercial se evidencia un problema: la dependencia exterior del hierro necesario para los flejes de barril, donde se envasa el vino para exportar. Sin embargo también se conoce la existencia de este metal en la serranía marbellí aún por explotar; hay cuatro minas en el término de Ojén, seis en Mijas, Benalmádena y Marbella. El hasta entonces comerciante malagueño empieza a dar los pasos necesarios para convertirse en industrial y en 1825 inscribe una mina a su favor; el siguiente es la cualidad empresarial que M. A. Heredia adquiere constituyendo la sociedad La Concepción con nueve socios que reparten ocho acciones cada uno. La constitución legal de la sociedad tiene lugar en 1830 y se inscribe en 
Málaga capital, donde consta en el Archivo Histórico Provincial (leg. 3929, fols. 21-39). Un hecho a destacar es que D. Owens vendió una mina de hierro de Ojén y un martinete, obtenidos por vía testamentaria de su tío G. O’Shea, a la sociedad La Concepción. De ello se deduce una tradición en la explotación de los veneros metálicos por extranjeros que se completa, y esto es el precedente en la producción férrica en Marbella, con la localización de un martinete accionado por la energía hidráulica del río Verde.

Otras minas de la reciente sociedad se localizan en El Peñón, El Peñoncillo y el Cerro del Sol (Marbella); la facilidad para explotar minas venía al amparo de la legislación minera favorable de la época fernandina. Para producir el hierro había que proveer combustible para los hornos de fundición; ya con anterioridad en 1826 el referido G. O’Shea había solicitado permiso para obtener leña del aprovechamiento del monte bajo de Propios, con destino a los hornos del martinete que laboraba el hierro sacado de su mina. Desde 1828 se suceden las solicitudes para explotar este recurso de Propios y, casi a la par, las denuncias y sanciones por el abuso en el monte de Las Chapas. En 1829 se describe la abundancia de ranchos carboneros, pero aún así se autoriza al director de La Concepción a disponer de las leñas de los montes de Sierra Real y Guerrizas de Marbella. Posteriormente las ferrerías de La Concepción y El Ángel arriendan al conde de Luque el monte bajo y los pinos de su jurisdicción, en el término de Benahavís, por plazo de 12 años; a partir de 1837 la Sierra Real está totalmente a expensas de La Concepción y de la contigua ferrería El Ángel, donde extraen leña para carbón. En 1846 P. Madoz censó en Benahavís más de un centenar y medio de habitantes dedicados exclusivamente a la arriería, conduciendo carbón de leña a las ferrerías del río Verde (datos del Archivo Municipal de Marbella y del Histórico Provincial de Málaga). Con ello se completa el segundo episodio de impacto
Vista del valle del río Verde: en primer término los restos de la antigua ferrería de La Concepción; al fondo, el embalse | foto Paisajes Españoles

Vista de los restos de los altos hornos y patio de molderías
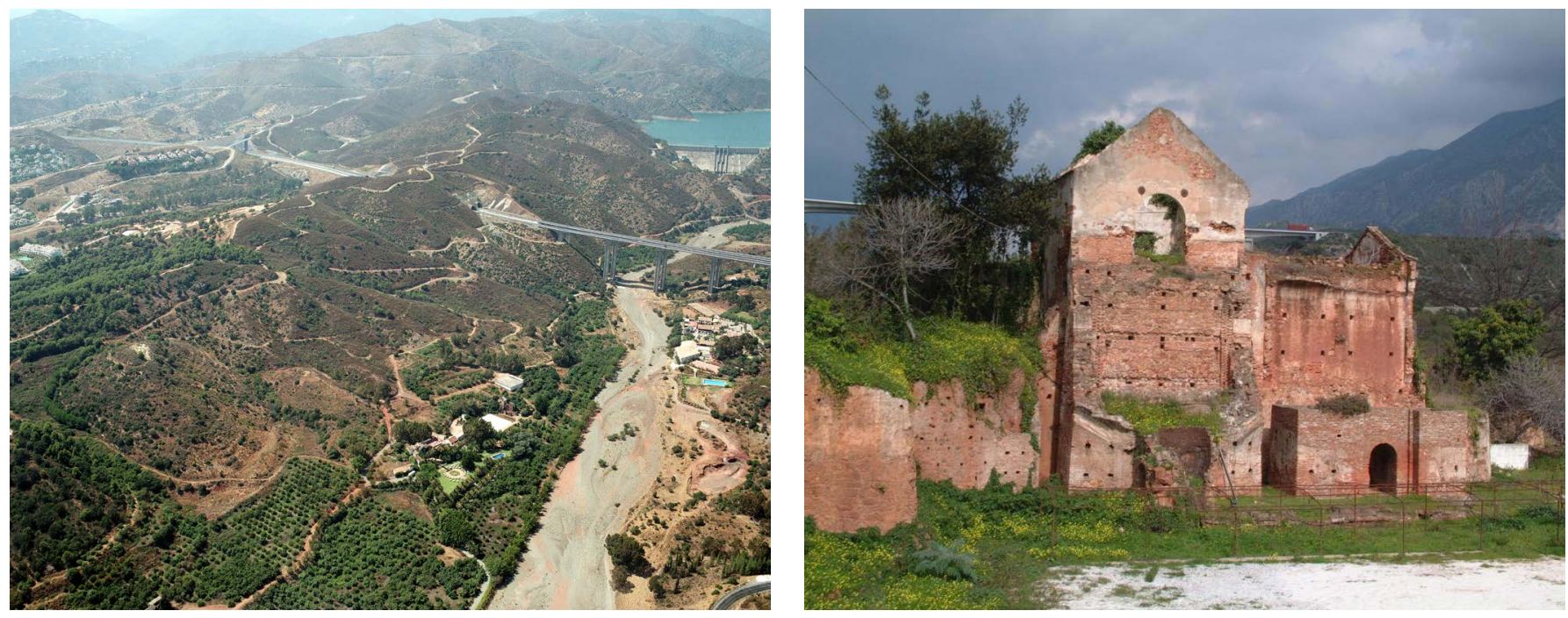
sobre la zona, si antes fue la extracción de grafito, ahora desaparecen los recursos forestales lo que fue denunciado por R. De La Sagra, J. M. de Nieva, Fermín Caballero, P. Madoz y, posteriormente, J. Costa.

Retrocediendo en el tiempo, en 1828, La Concepción, sociedad inscrita en 1826, inicia la producción con el sistema de forjas "a la catalana" (farga), con ingenieros y fundidores piamonteses y operarios belgas y franceses. La energía hidraúlica la proporcionan dos ruedas de veinticinco y catorce caballos, a las que se unirá en el futuro una de ocho y todo el combustible que proporciona el carbón leña de los bosques. El personal lo componen 1.084 hombres ayudados por 836 acémilas. Hasta 1830 esta era la situación en el complejo productivo que realmente no proporcionaba resultados satisfactorios a causa de que el mineral de Ojén presentaba dificultades por su composición, altamente refractaria, lo que producía una díficil fundición por resistencia al calor de los hornos bajos. Este período experimental de aciertos, combinado con errores, supuso un aumento de los gastos y un temor entre los socios que pronto se desprendieron de sus acciones quedando $\mathrm{M}$. A. Heredia solo al frente de la fundición. Este es el momento de inflexión que hará que se transforme el complejo con tecnología diferente y una planificación especial.

El empresario se asesora por el comandante Francisco Antonio Elorza, ingeniero exiliado después del trienio liberal (1820-23), que desde su marcha en 1823 hasta su vuelta en 1828 recorre las fundiciones de Inglaterra, Bélgica (sobre todo Lieja), Hortz (Alemania), Piamonte (Italia) y Francia, estudiando

Antiguas dependencias de la ferrería: acceso a la capilla y abrevadero de acémilas de la antigua factoría, hoy convertidos en residencia
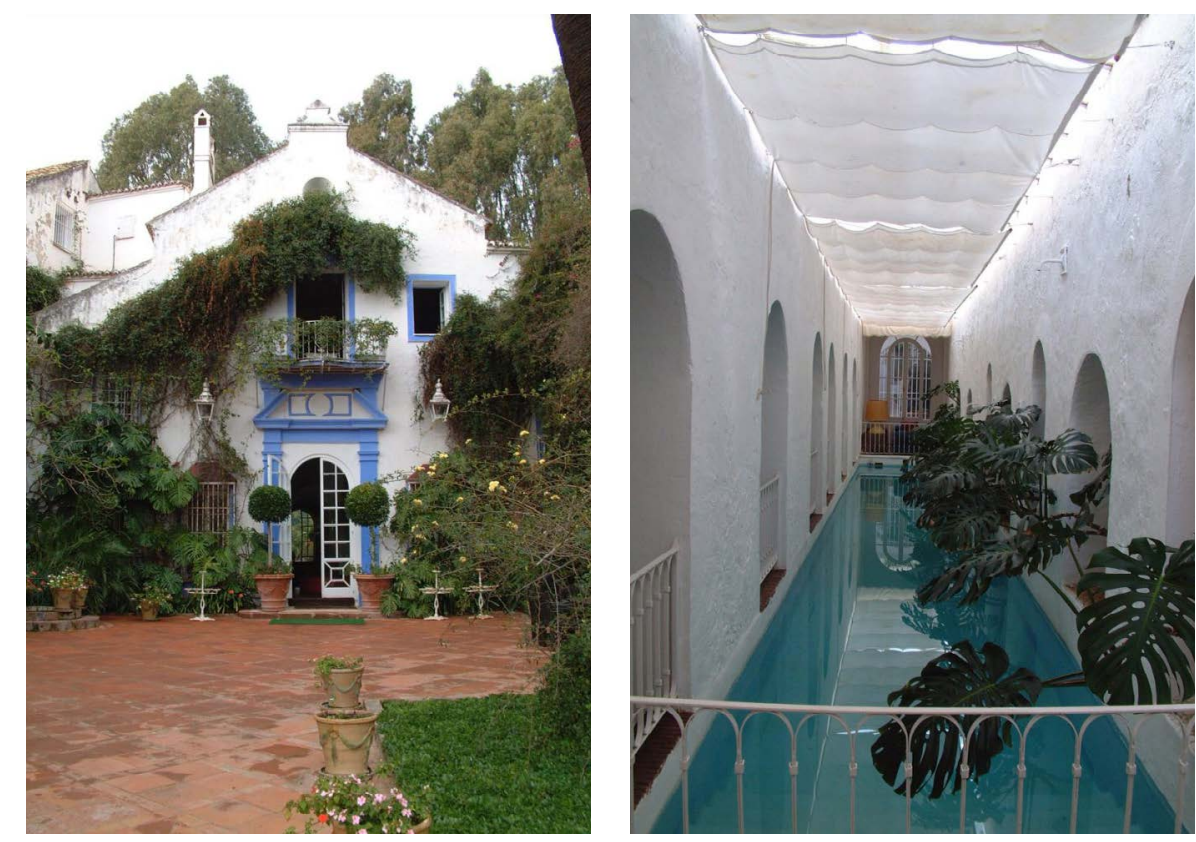
sus métodos y completando su formación como ingeniero. El resultado consiste en cambiar las forjas "a la catalana" por forjas "a la valona", y en vez del metódo directo para obtener el hierro dúctil, el indirecto de altos hornos ingleses o de puddler para afinar los lingotes, usando como combustible el carbón de piedra para el pudelado. De esta forma, en 1832 se encienden por primera vez los nuevos altos hornos, inaugurando una etapa que durará hasta 1838 y un epílogo hasta 1843; ya desde 1836 evidencian los nuevos problemas con los que se había hipotecado el procedimiento iniciado en 1832.

A la ferrería de La Concepción se accede a través de la carretera de Marbella a Istán, situada junto a la margen derecha del río Verde, el cual le proveía de energía hidráulica mediante un canal que conectaba aguas arriba. El conjunto constituye un interesante modelo de arquitectura industrial; de la instalación se distinguen los restos de tres altos hornos situados en un desnivel y, separados de ellos, restos de las cuadras y abrevaderos para acémilas de carga, junto a otras dependencias convertidas hoy en residenciales. Abarcando el conjunto y a una cierta distancia se sitúa parte de la cerca que delimitaba el conjunto donde se conservan los restos del acceso. La pendiente natural hacia el río se aprovecha excavando un desnivel artificial de unos seis metros donde se sitúan los hornos, lo que facilita su alimentación arriba y colada abajo; están conectados con la cota de llegada del mineral a través de un pequeño viaducto con arcos que nivela cota de acceso desde la entrada con la edificación, hoy en alberca, que conecta con el tragante del horno. En la parte inferior se sitúan las bocas de colada y de insuflado de aire que aviva el horno mediante barquines, según

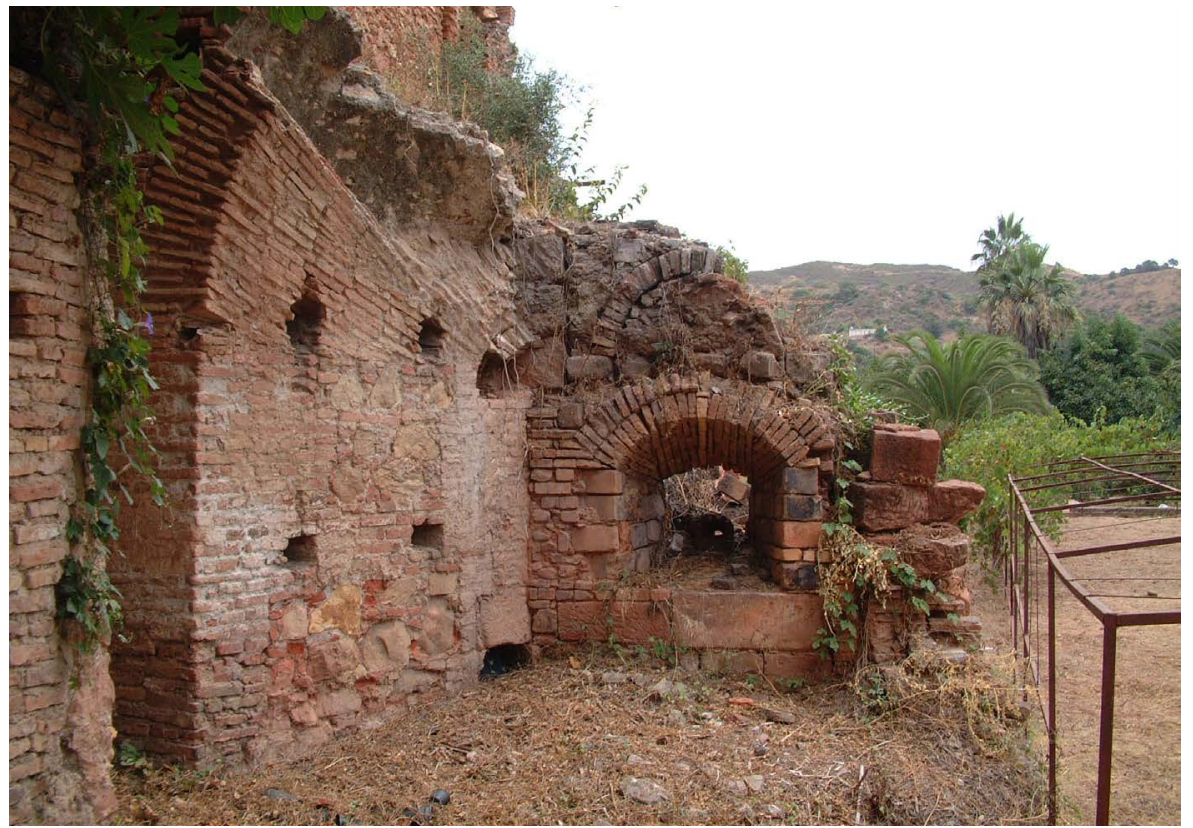

Restos de la bóveda occidental del alto horno Conde; se aprecia una de las boquillas de insuflado de aire en el crisol a través de barquines 


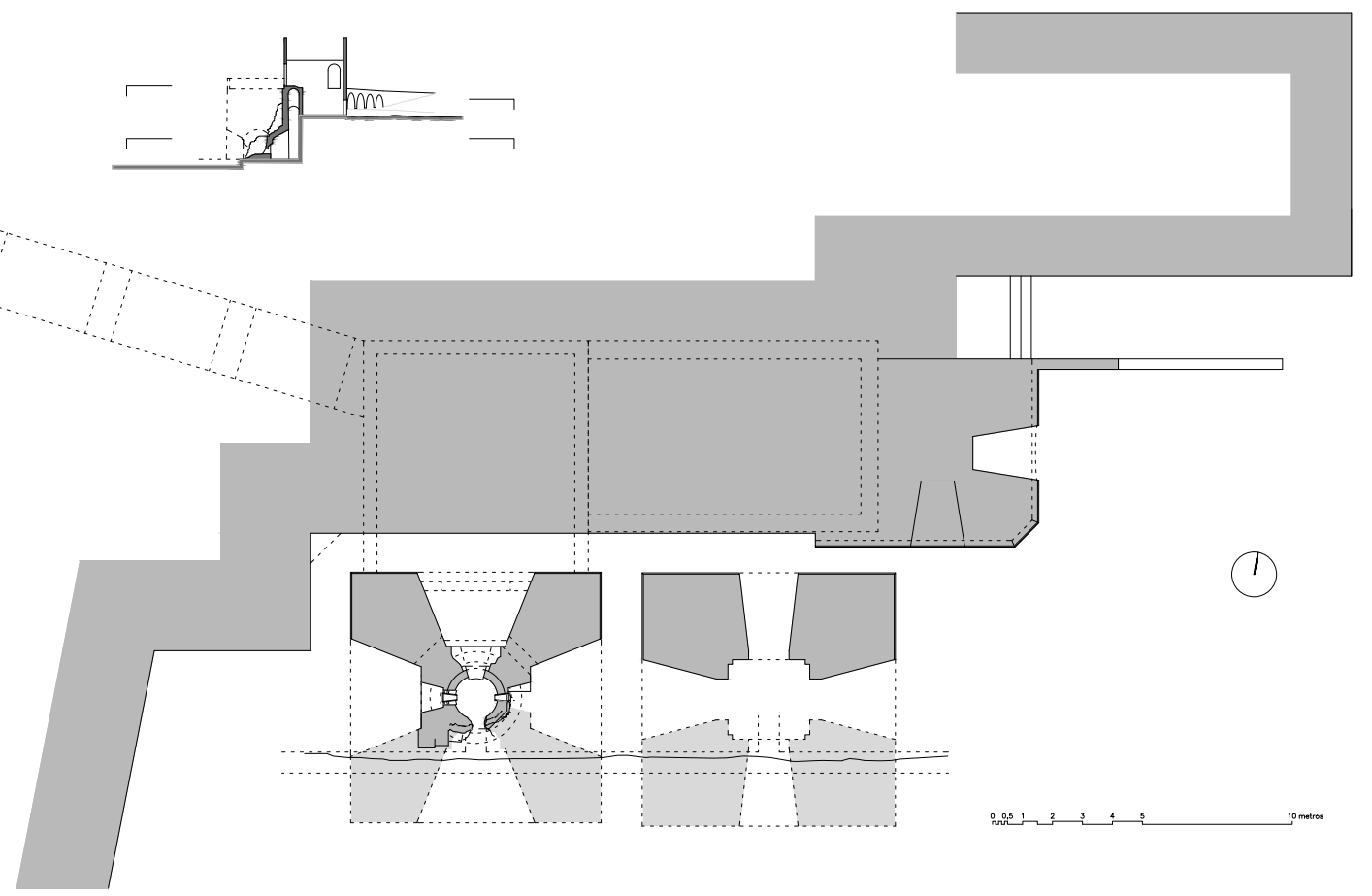

Planta de la zona de hornos (reconstruidos en color claro)

un esquema clásico de planta cuadrada con bóvedas de registro por cada cara. La cuba del horno está ejecutada con ladrillo refractario de origen inglés con distinto aplantillado; el núcleo constructivo se reviste con un forro exterior de fábrica en forma tronco-piramidal, zunchado a distintos niveles con barras pasantes de acero provistas de traviesas en sus extremos. Aunque del mejor conservado sólo queda parte de la base y de la cuba, se encuentra una prolija descripción (véase página 82) en Naranjo y Garza (1844) que ha permitido su reconstrucción formal. El resto del espacio de lo que fue el patio de molderías se encuentra expedito y se aprecian mechinales y restos de tinglados fabriles en el muro de contención que construye de desnivel con el terreno natural.

Los problemas previstos en seguida mutaron a evidentes: desde 1832 era fijo el hierro colado que abastecía horno de afino y forjas, y el carbón-leña de pinos y arbustos que se quemaba en las forjas a la valona y en los altos hornos; sin embargo para el pudelado había que traer del exterior carbón hulla, asturiano o inglés. Ello aumentaba los costos que se incrementaban con el transporte de la hulla desde el puerto de Marbella hasta la factoría; de nuevo la dependencia del exterior, en este caso de combustible, lastraba la producción andaluza. En 1833 se trasladan las operaciones de afino a una nueva factoría, establecida en Málaga capital (playa del Carmen), inaugurada como sociedad La Constancia. En 1836 esta factoría reducía el lingote a hierro forjado mediante 7 afinerías en hornos pudler recalentados 


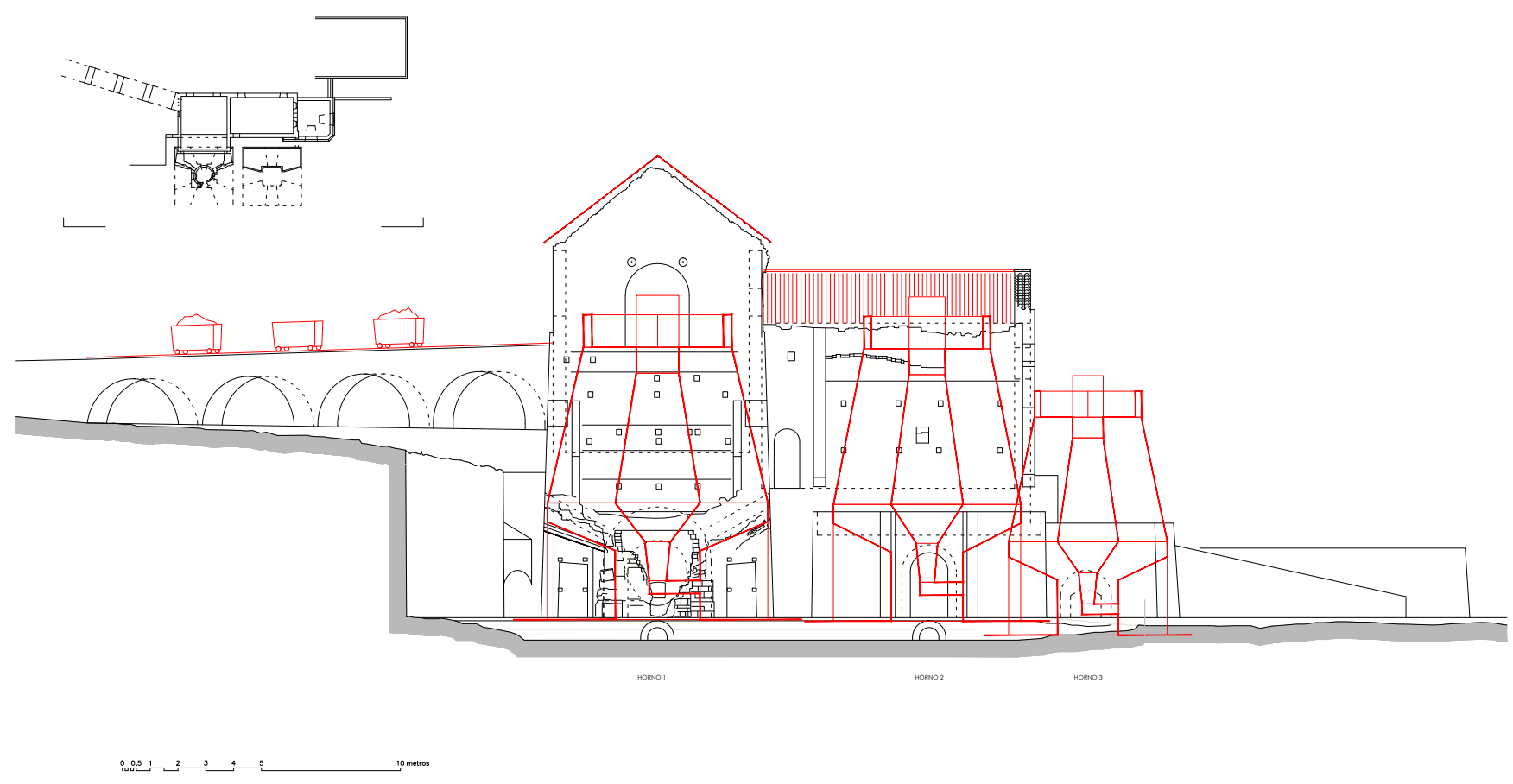

Alzado sur de los restos de altos hornos (reconstruidos en rojo) | dibujos e hipótesis reconstructivas de los

mediante otros tres hornos. Una máquina de vapor de $30 \mathrm{cv}$ de potencia aliautores mentaba el tren de cilindros, martillo y tijeras.

A partir de 1843 la siderurgia de La Concepción trató inútilmente de usar tan sólo antracita (carbón mineral) y M. A. Heredia adquiere maquinaria inglesa. La operación no dio resultado y a partir de ahí se funde casi exclusivamente en La Constancia; los fletes de transporte de carbón encarecían este si había que trasladarlos a Marbella y hacía más barato que todas las operaciones se radicasen sólo en Málaga capital. La ferrería marbellí de Heredia había cambiado de propietario y, hacia 1885, aparece como uno de los títulos de la sociedad Altos Hornos de Málaga, constituída legalmente en 1899 en Bruselas con 35.000 acciones (100 francos por acción).

Perdida la actividad fundidora, no sucede lo mismo con la extracción de metal que ahora es explotado por la sociedad Marbella Iron Ore Limited; esta compañía, a través de su apoderado, súbdito inglés William Malcom, y de su apoderado español Miguel Calzado Martínez, presenta al Ayuntamiento de Marbella un proyecto de construcción de un muelle de hierro para embarque del mineral extraído de la mina El Peñoncillo, antigua propiedad de Heredia. El muelle, según plano de 1869, tendría 344 metros de los que 281 discurrían dentro del mar; con posterioridad la terminal se ensancha en su extremo y dispone de una doble vía por donde retornan los vagones, ya descargados, empujados por los que vienen de ida, cargados. A la continuidad 
Axonometría del conjunto con la reconstrucción de dos altos hornos | dibujo e hipótesis reconstructiva de los autores

Reconstrucción geométrica de un alto horno de la Ferrería según la descripción de Felipe Naranjo y Garza | dibujo Óscar Gil Delgado

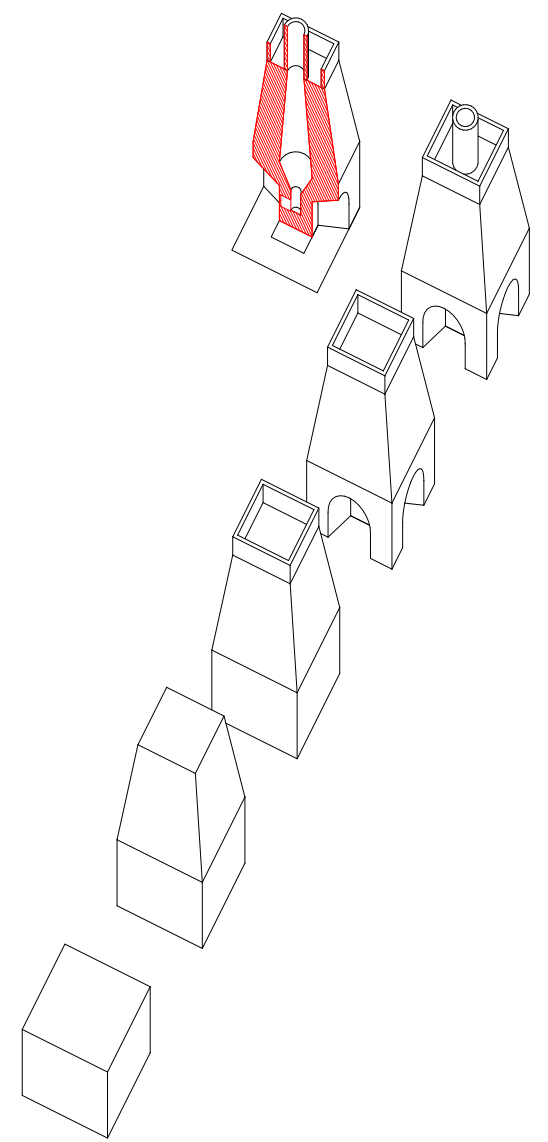

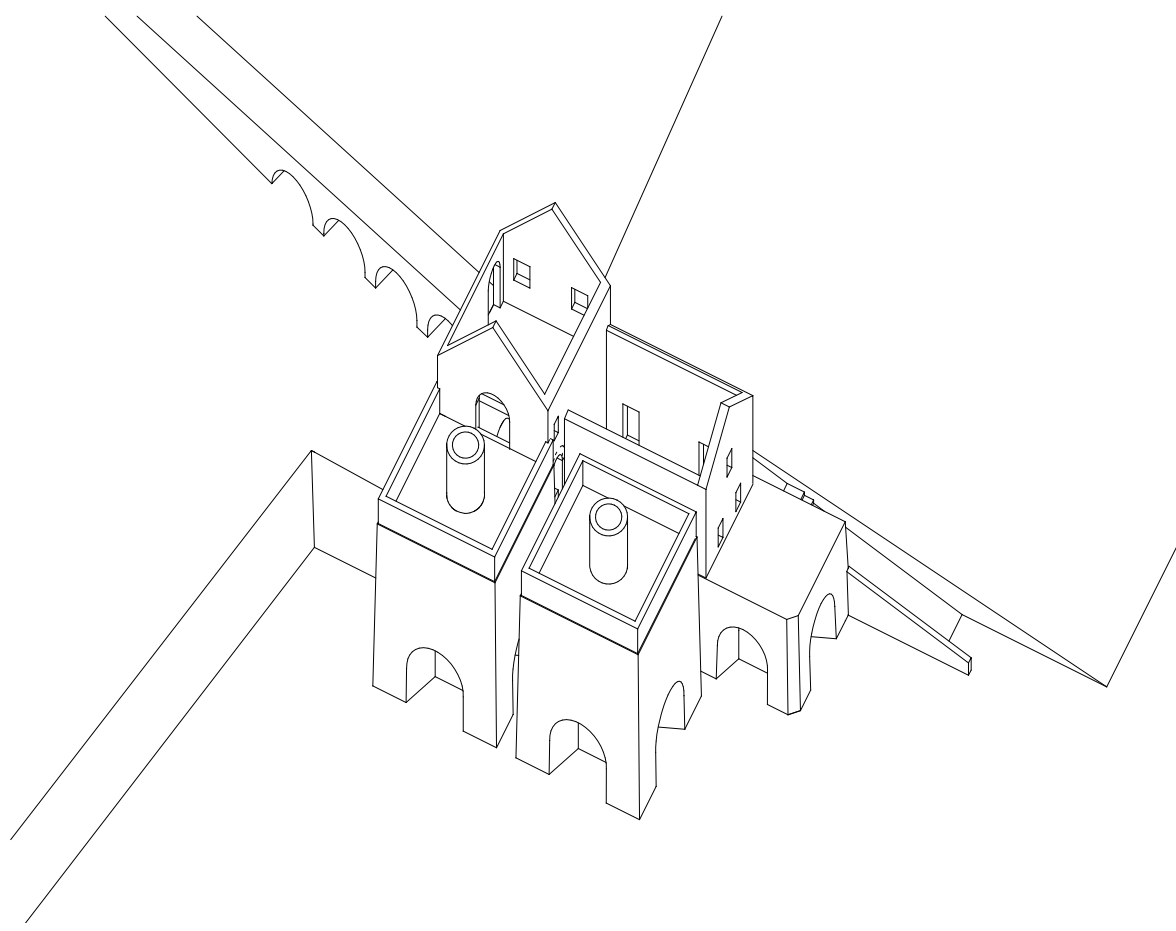

"Se forma un muro de ladrillo ordinario o macizo de 26 pies en cuadro y 18 de elevacion, de figura prismatica, cuyo macizo se eleva otros 24 pies mas en la de piramide truncada, teniendo 17 y medio pies en cuadro en la parte superior. Sobre este macizo esta construida una barandilla o pretil con paredes sencillas de 5 pies de altura guamecidas a veces de almenas para resguar-do de los cargadores. La obra toda esta asegurada por fuertes llaves o barras de hierro forjado y en la parte inferior, o sea, en la delantera del homo hay una apertura para la sangria o colada y por cuya abertura dirigen los operarios con sus espato-nes y otras herramien tas la marcha de la produccion; otras dos aherturas laterales algo mas pequefias sirven para conducir el viento al homo por medio de las correspondientes toberas, las cuales se hallan casi horizontales o con muy escasa inclinacion hacia arriba por requerirlo asi el punto de fusion de esta clase de menas. En el interior del macizo esta construido el homo pro-piamente dicho, en donde se reduce el mineral y como todos los de su clase se compone de crisol, artalajes y cuba, y esta cuba tiene 26 pies de elevacion, 10 pies de diametro en su par-te inferior o vientre del homo y 4 pies 10 pulgadas en la parte superior o tragante, desde cuyo punto arranca la chimenea que tiene 12 pies de altura. El crisol afecta la figura de una pira-mide truncada inversa con 20 pulgadas en cuadro en la parte inferior y 33 pulgadas en la superior y la union de esta parte del homo con la cuba que forman los atalajes es como un cono truncado de 6 pies de elevacion. El crisol que tambien tiene 6 pies de altura se prolonga en su parte inferior hacia el frente de la colada de modo que presenta un rectangulo de 5 pies y 10 pulgadas de largo y 1 pie y 8 pulgadas de ancho que es el punto en donde se reunen el metal fundido y las escorias que por su ligereza o menor gravedad especffica ocupan la parte superior; resulta pues, en suma, que el homo descrito viene a tener unos 48 pies de elevacion. Para la construccion del crisol se emplea una arenisca del termino de Marbella, fina, granuda y resistente al choque (...) y los ladrillos refractarios para la cuba, chimenea y atalajes vienen de Inglaterra." (NARANJO Y GARZA, 1844: 46) 


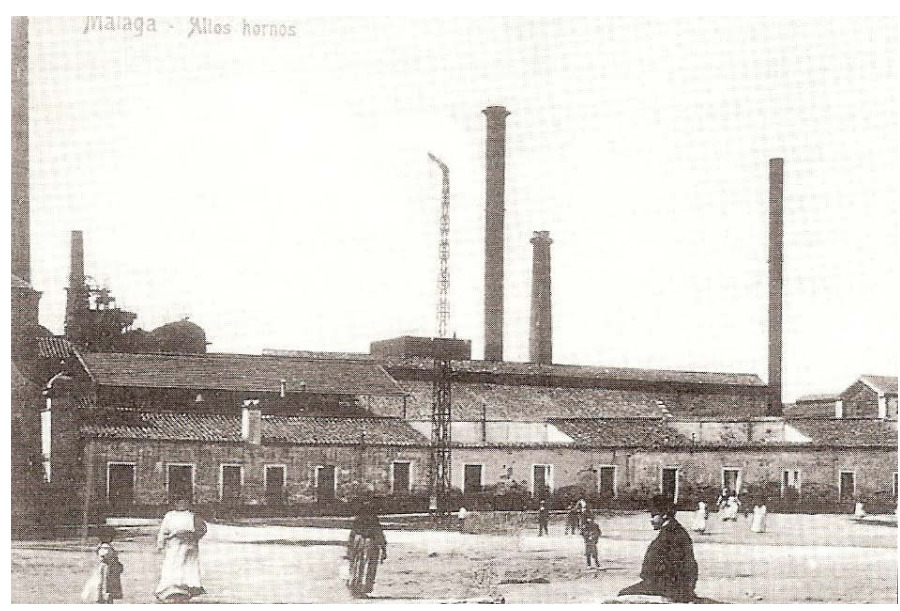

de la explotación minera se añaden transformaciones con infraestructuras como la construcción de este muelle metálico en la rada marbellí, y un proyecto de ejecución de tren minero y vía (el llamado tren San Juan Bautista).

La disociación extracción-transformación marcan el final de la explotación fundidora e inauguran una fase de transformaciones del territorio, con obras de infraestructura en el puerto viejo, trazado del ferrocarril minero y cambios urbanísticos en el casco histórico. William Lonsdale, socio del ferrocarril, propone al Ayuntamiento la cesión mediante Real Venta de 5.074 metros cuadrados en el centro urbano de Marbella con la finalidad de construir almacenes, oficinas y las terminales férreas en dos ramales: el del muelle y el de almacenes de custodia y reparación. Con esta operación se sustituye el antiguo y obsoleto "Corral del Hierro", almacén de época de Heredia, de cara a la nueva realidad industrial y minera. La operación es rematada con el pago de 14.270 reales y una serie de medidas compensatorias para el municipio.

La Marbella Iron Ore Limited explotaba la mina de El Peñoncillo organizando el filón en nueve niveles junto a otros criaderos de nueva propiedad y, hacia 1898, extraen 25.053 toneladas de mineral-hierro, que ya no se transforma, con una ganacia de 5.144 libras (4,87 libras por tonelada, según la Revista Minera, Metalúrgica y de Ingeniería de 1899).

Hasta aproximadamente 1934 la compañía inglesa continuó trabajando y, en torno a esos años previos a la guerra civil, la explotación se abandona y es desmantelada; igual suerte corre el muelle de carga que es desmontado y vendido como hierro. Tras la contienda española se vuelven a aprovechar los escoriales de El Peñoncillo; hay que recordar que este hierro contiene altos índices de pirrotina (rica en azufre) lo que hace que parte de lo explotado por los ingleses careciese de valor y quedase abandonado en voluminosos escoriales. 
La empresa vasca Ferarco, S. A., en la década de los 60 del siglo XX, explota los escoriales arrastrádandolos mediante cucharas y transportándolo en camiones hasta una planta de concentración donde era machacado y, con separadores imanadores, se extraía el hierro; se transporta mediante un cable con cubas que se vacían sobre barcos en el mar.

Hacia finales de siglo XIX la visión de Marbella es la de un término que ha soportado el primer ensayo de altos hornos tipo inglés en España y más concretamente Andalucía y Málaga. En ese camino existen grandes logros, como la alta posición en la producción férrica española, y un costo, el esquilme de recursos mineros como el grafito y el mineral de hierro naturales así como el bosque y arboleda mediterráneas y que acarrea transformaciones terriroriales de infraestructuras y urbanísticas como el trazado del ferrocarril, el propio ferrocarril, el muelle de hierro y los almacenes que sustituyen a los de Heredia. 


\section{BIBLIOGRAFÍA}

- ÁlVAREZ de LINERA, A. (1857) Memoria histórica, científica y estadística sobre las minas de grafito del partido de Marbella pertenecientes al Estado. Revista minera, T. VIII, 1857, pp. 648-656; 704-713

- BLANQUI, G. A. (1850) Rapport sur la situation economique et morale de l'Espagne en 1846. París, 1850, p. 39

- CHASTAGNERET, G. (1974) La legislation de 1825 et l'evolution des activites minieres. En Agricultura, comercio colonial y crecimiento económico en la España contemporánea: Actas del Primer Coloquio de Historia Económica de España (Barcelona, 11-12 de Mayo de 1972). Barcelona: Ariel, 1974

- DEBARY, TH. (1849) Travels in the South of Spain, Algiers and Canary Islands. 1849

- ORTUETA Y DUARTE, D. DE (1919) Informe sobre el reconocimiento de la Serranía de Ronda. Madrid: Sucesores de Rivadeneyra, 1919

- EZQUERRA DEL BAYO, J. (1846) Sobre una memoria publicada por M. Pernolet con el título de Note sur les mines et les fouderies du midi de I' Espagne. Por D. Joaquín Ezquerra del Bayo [sic]. Anales de minas, T. IV, Madrid, 1846, pp. 507-513

- GARCía MONTORO, C. (1977-78) Fundación de las ferrerías "La Concepción" y "El Ángel" de Marbella. Anuario de historia contemporánea, 4-5, 1977-78, pp. 285-293

- GARCíA MONTORO, C. (1979) La Siderurgia de Río Verde y la deforestación de los montes de Marbella. Moneda y crédito, 150, 1979, pp. 79-95

- GoYANES, I. (1841) Descripción razonada del procedimiento que sigue para el afino del hierro colado por método inglés en la herrería nombrada de la Concepción, en las inmediaciones de Marbella. Anales de minas, T. II, 1841 , pp. 359-394

- SAGRA, R. DE LA (1845) La Herrería del Sr. Heredia en Málaga. Extracto de un viaje a las costas de Andalucía. Boletín de empresas dedicado al mayor y más rápido acrecentamiento de la riqueza pública, I, n.o 23 (30. IV.1845), pp. 181-183

- LE PLAY, F. (1834) Itineraire d'un voyage en Espagne. Procédé d'un aperçu sur l'etat acuel et sur l'avenir de I'industrie minerale dans ce pays (20 avril-15 luillet 1833). Annales des mines, $3^{\mathrm{a}}$ serie, T. V, París, 1834

- MARTÍNEZ Y MONTES, V. (1852) Topografía médica de la ciudad de Málaga. Málaga: Círculo Literario, (Imp. de D. Ramón Franquelo), 1852, cap. XI

- MADOZ, P. (1947) Diccionario Geográfico-EstadísticoHistórico de España y sus posesiones de ultramar. T. 7.
1847

- MENÉNDEZ PIDAL, G. (1988) La España del siglo XIX vista por sus contemporáneos. Madrid: Centro de Estudios Constitucionales,1988, p. 337

- NADAL I OLLER, J. (1972) Industrialización y desindustrialización del sureste español 1817-1913. Moneda y crédito, n. ${ }^{\circ} 120,1972$, pp. 3-80

- NADAL I OLLER, J. (1974) El fracaso de la Revolución Industrial en España 1814-1913. Barcelona: Ariel, 1974

- NARANJO Y GARZA, F. (1844) Minas de hierro y ferrerías de Marbella. Boletín oficial de minas, T. I, 15 de junio de 1844 , p. 46

- PERNOLLET, M. (1854) Notice sur les usines à fer de Malaga suivie de considérations relatives au traitement des minerais de fer en Algérie. Annales des mines, $4^{\mathrm{a}}$ serie, t. VIII, 1854, pp. 595-634 (p. 596)

- RUBIO DE ARRIBA, V. (1820) Estructuras Industriales. DOSSAT 1968. Fig. VI- 31: Restos (1960) del horno alto levantado en Marbella (Málaga) el año 1829

- SALES GARCíA, F. (1841) Sobre las minas y fundición de Marbella. Forma parte de una memoria presentada à la Dirección general de minas, por el ingeniero del distrito $D$. Francisco Sales García en 1873 [sic]. Anales de minas, Vol. II, 1841, pp. 347-358

- WALIS, S. T. (1849) Glimpses of Spain, or Notes of an Unfinished Tour in 1847. N. York, 1849, pp. 86-89

- POLLARD, S.; HOLMES, C. (1868) Documents of European Economic History: Vol. I. The Process of Industrialization, 1750-1870. London, 1868, pp. 362-363 